\title{
Paediatric Dissecting Aneurysm of the Posterior Cerebral Artery Case Report and Review of the Literature
}

\author{
G.B. BRADAČ, P. PERETTA*, G. STURA, P. RAGAZZI*, P.P. GAGLINI*, M. BERGUI \\ Neuroradiological Department, Molinette Hospital University of Turin \\ *Neurosurgical Department, Paediatric Hospital Regina Margherita; Turin
}

Key words: intracranial aneurysms, children, dissection, endovascular treatment

\section{Summary}

Aneurysms in children are rare. We describe a large spontaneous dissecting aneurysm of the posterior cerebral artery. The clinical presentation was characterized by headache as the sole symptom due to a mass effect leading to hydrocephalus. Acute treatment with a temporary ventricular shunt was followed by occlusion of the aneurysm via an endovascular approach leading to a complete recovery of the patient.

\section{Introduction}

Cerebral aneurysms in children are relatively rare with an incidence ranging from $0.5 \%$ to $5 \%{ }^{1-6}$. There are some differences in the aetiologies, localization and clinical presentation in comparison to aneurysms in adults. We describe a case with the clinical aspects, diagnosis and treatment.

\section{Case Report}

A seven-year-old boy with a three day history of severe headache was seen in the out-patient's department of the Paediatric Clinic. Clinical examination failed to disclose neurological or other pathological signs. There was no trauma in the clinical history. Infections, systemic collagenous diseases, haemoglobinopathies and familial history for aneurysms were excluded. CT and MR scans showed a mass, probably a partially thrombosed aneurysm of the posterior cerebral artery, compressing the mesencephalon and aqueduct leading to mild dilatation of the IIIrd ventricle and lateral ventricles (Figures 1 and 2). There were no signs of subarachnoid hemorrhage. The patient was admitted to the Neurosurgical Department and a few days later the patient was referred to the Neuroradiological Department for an angiographic examination.

The angiogram showed a large irregular partially thrombosed aneurysm supplied by a branch arising from P3-P4 segment of the posterior cerebral artery. There was a focal stenotic segment proximal to the aneurysm. These findings where suggestive for a dissecting aneurysm (Figure 3A). A preventive external ventricular shunt was performed and the headache improved gradually.

A multidisciplinary decision was taken to occlude the aneurysm and endovascular treatment was planned and performed a week later. Under general anesthesia and full heparinization via the femoral artery a $5 \mathrm{~F}$ guiding catheter was placed in the right vertebral artery and a microcatheter (Excelsior SL10, Boston Scientific Target) was advanced into the right posterior cerebral artery.

The aneurysm was catheterized and occluded along with the distal segment of the parent artery with coils (Figure 3B,C). 


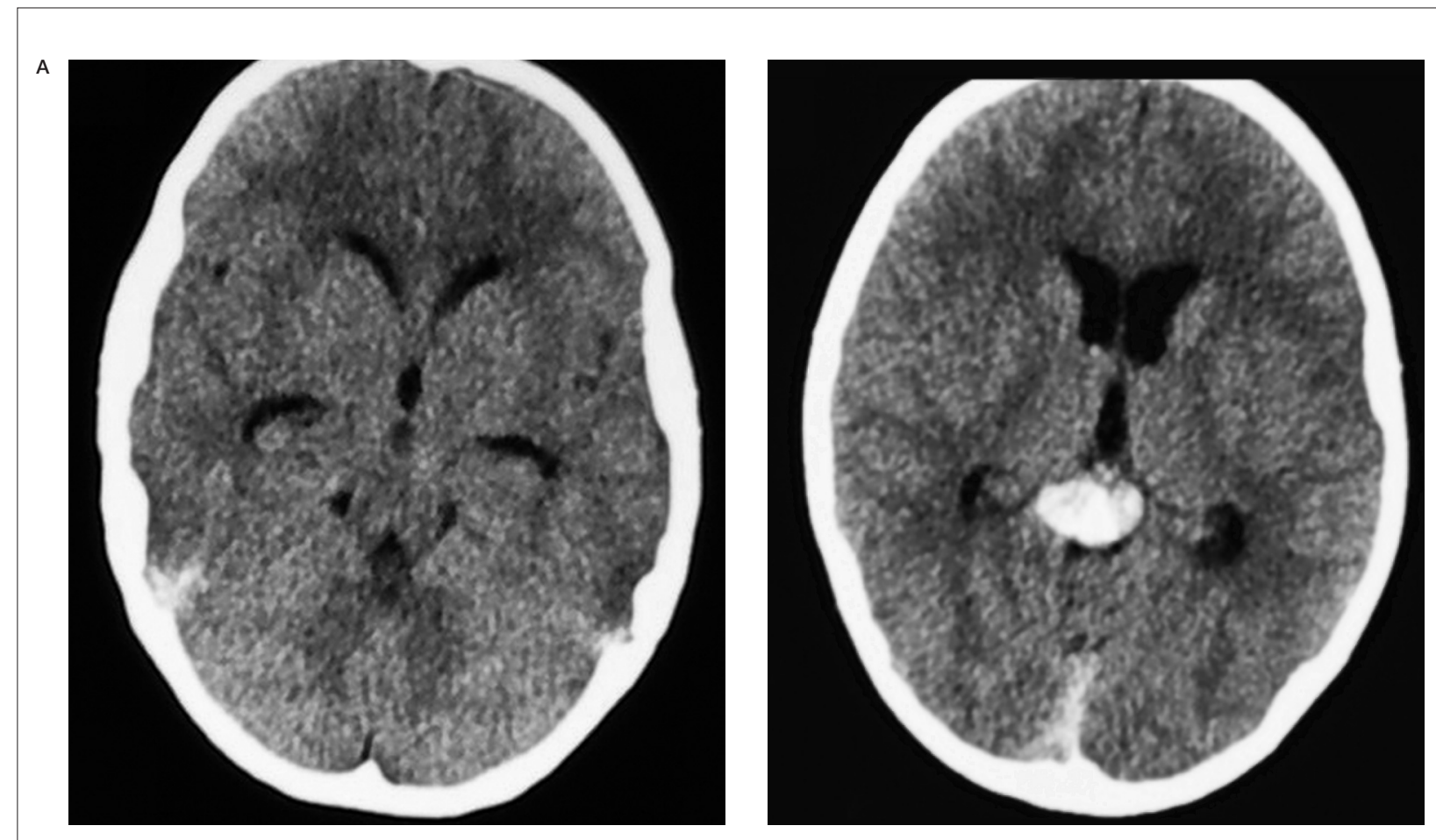

Figure $1 \mathrm{CT}$. There is a disomogeneous hyperdense round lesion compressing the aqueduct and mesencephalon leading to mild hydrocephalus.

The patient tolerated the procedure well without any neurological deficit. Probably the compression of the aqueduct decreased rapidly following thrombosis of the aneurysm so that the ventricular shunt was no longer necessary. The patient, free of symptoms, was discharged ten days later. MR scan (Figure 4) and angiography performed six months later confirmed the disappearance of the aneurysm.

\section{Discussion}

Intracranial aneurysms in children differ from those in adults in the localization, aetiology and clinical presentation. They are more common in boys ${ }^{2,3,7,8}$ whereas in the adult there is a predominance in women. This seems indicate a gender influence.

The most common site of paediatric intracranial aneurysms, as reported in the literatu$\mathrm{re}^{1,2,3,7,8}$, is the internal carotid artery (cavernous portion, carotid bifurcation). Aneurysms in the posterior circulation are less frequent, however their incidence in this sector is much higher than in adults ${ }^{3,7}$.

At the time of diagnosis, the aneurysms are often large or giant with clinical symptoms frequently not due to hemorrhage but to mass effect on the adjacent structures causing neuro- logical symptoms and headache. Headache was present as an isolated symptom in $25 \%$ of the series of Lasjaunias ${ }^{3}$. Headache was the sole symptom of our patient in particular due to the peculiar localisation leading to aqueductal stenosis and mild hydrocephalus. Aneurysm is an exceptional cause of triventricular hydrocephalus in children. In the clinical differential diagnosis, infections, hemorrhage, CNS malformations, tumours, aneurysm of the vein of Galen, and midbrain arteriovenous malformations crossing directly or by draining veins should be considered ${ }^{9-13}$.

The causes of aneurysms in adults have extensively been discussed. Microscopic studies on the wall of the aneurysms have shown fragmentation or absence of both the internal elastic membrane and muscularis layer of the media at the V-shaped distal division of bifurcations. This can be a congenital defect or more probably ${ }^{14,15}$ an acquired degenerative process as a consequence or facilitated by many factors (high blood pressure, stress forces, atherosclerosis, smoking). All these elements finally lead to the formation of the aneurysm in the adult.

Unlike adults, a frequent cause of aneurysms in children is trauma, and in this context previous even minor head traumas could be of importance. The incidence of post-traumatic aneur- 


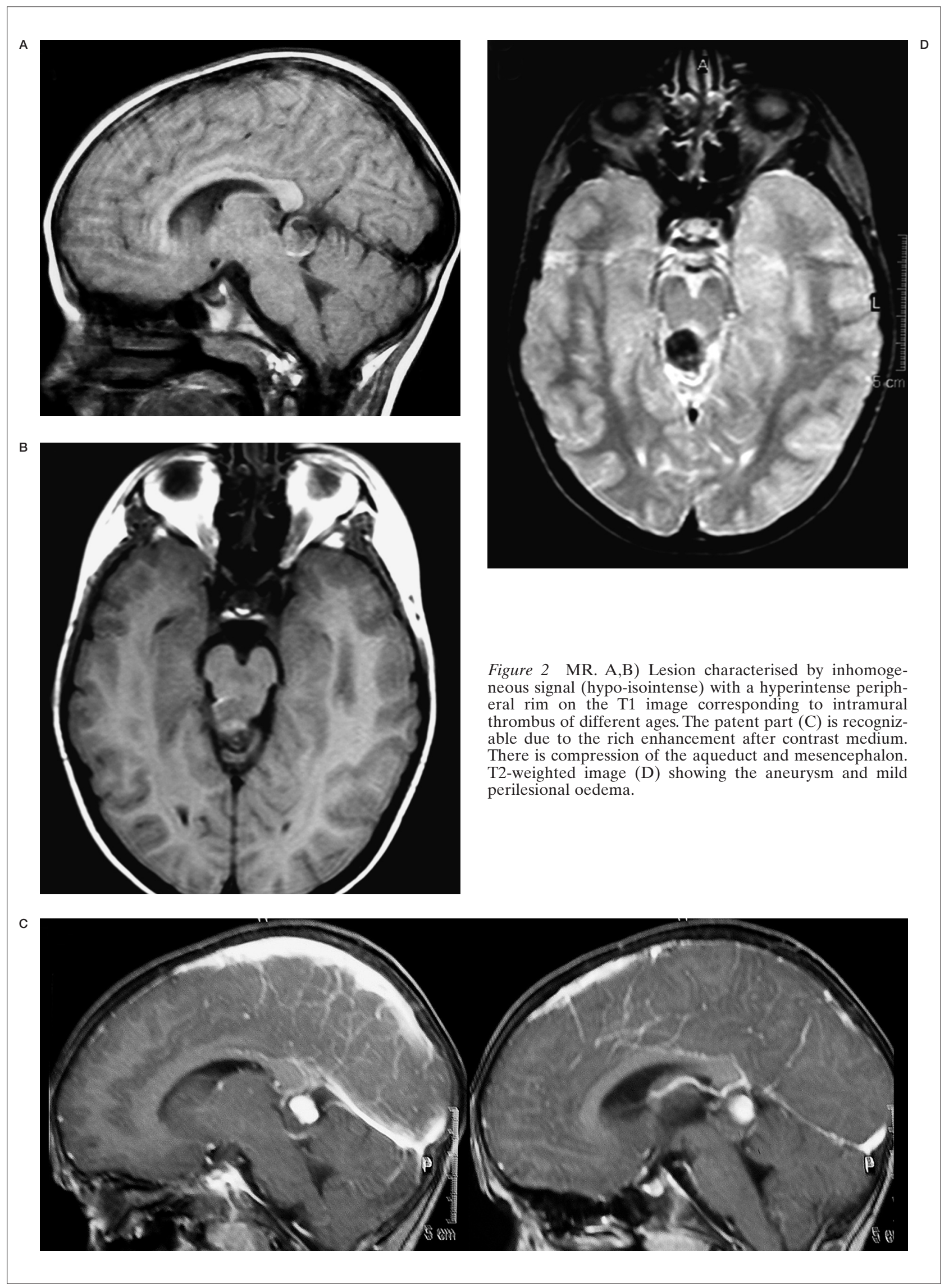



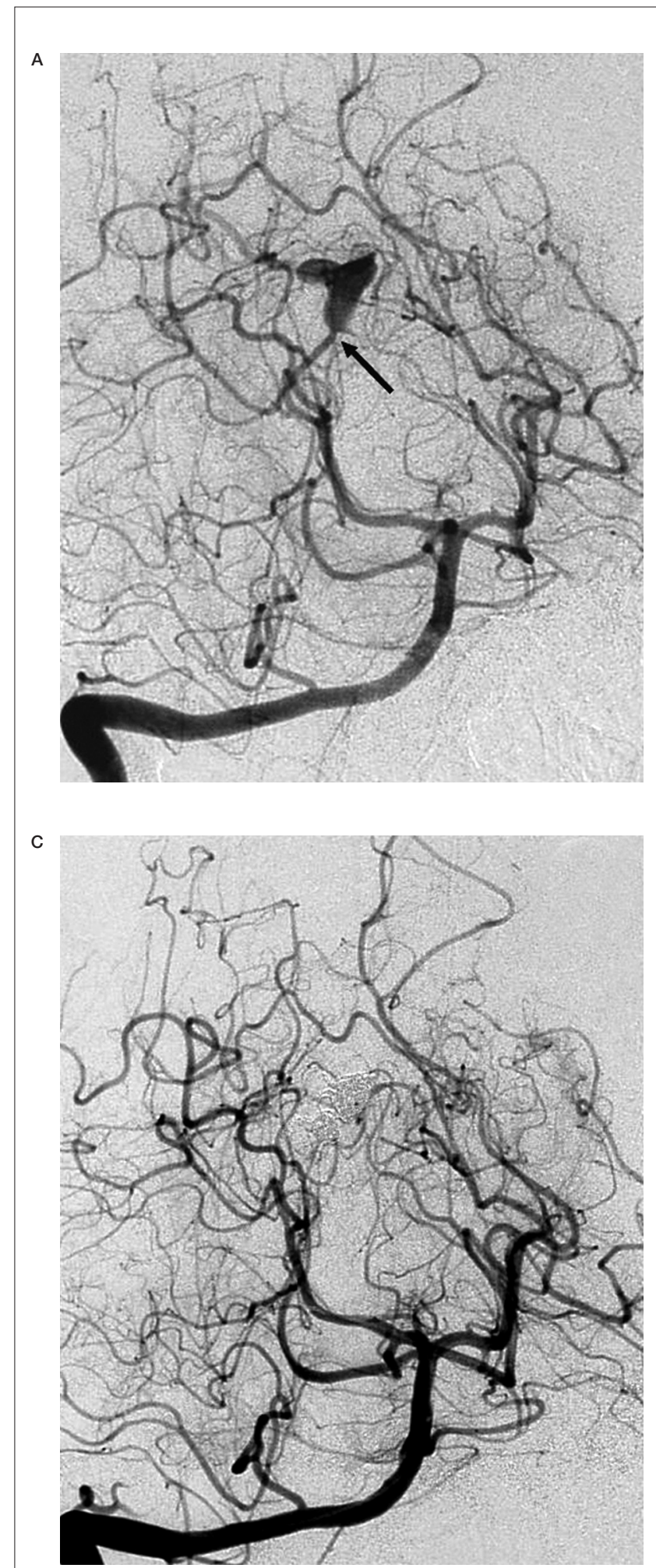

Figure 3 On the angiogram (A) an irregular shaped aneurysm arising from a small branch of the P3-P4 segment of the PCA is visible. There is a focal stenotic segment proximal to the aneurysm (arrow) Selective catheterisation of the aneurysm occluded with coils along with the small supplying branch (B). Final control angiogram after treatment (C).

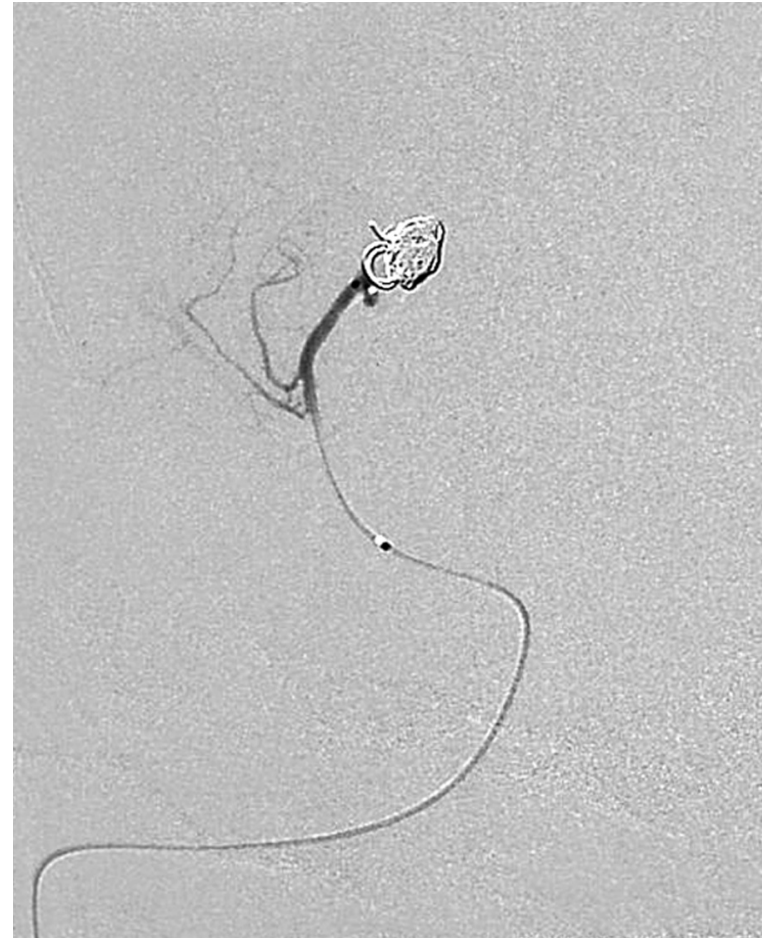

ysms as reported in the literature varies from $10 \%$ to $39 \%{ }^{7,16,17}$. Other causes are infections, systemic collagenous diseases, hemoglobinopathies as well as familial history of aneurysms $1,3,4,5,6,8,18$. More recently, spontaneous dissection has been increasingly recognized as a cause of cerebral aneurysm in children ${ }^{3,7,19,20,21,22}$. Moreover in the reports of some authors ${ }^{3}$ the incidence of dissecting aneurysm is particularly high in aneurysms of the posterior circulation. In this context aneurysms of the posterior cerebral artery are relatively common ${ }^{3,7,19,22}$. In our case the localization of the aneurysm and its MR and angiographic features were highly suggestive of a dissecting aneurysm. The headache was due to the mild hydrocephalus and probably also to the acute onset of an intramural hemorrhage.

In non ruptured dissecting aneurysms it is not always easy to decide which kind of therapy is the most suitable: conservative or more aggressive. Indeed, cases of spontaneous thrombosis with disappearance of the aneurysm in the follow-up have been reported ${ }^{22}$.

On the other hand we know that the risk of bleeding, which can have catastrophic consequences, is very high in dissecting aneurysms, and so we decided in our patient for a more aggressive therapy. 

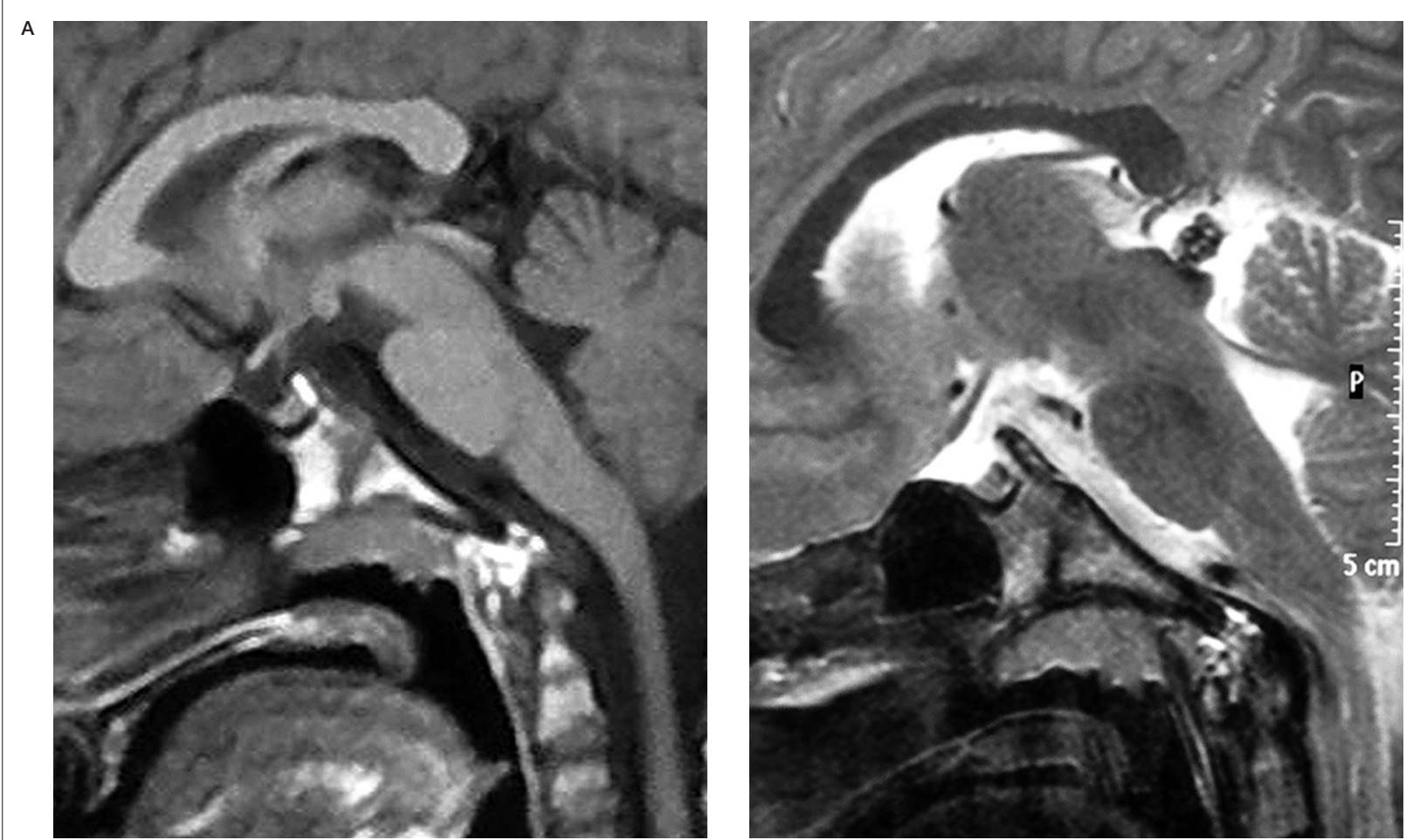

Attention should be paid to large-giant partially thrombosed aneurysms. This is a particular situation already the object of study in the past ${ }^{23}$ and again considered more recently ${ }^{24}$. In many of these cases we are probably dealing with dissecting aneurysms in which the thrombus is not located in the lumen but within the wall of the aneurysm. This leads to an inflammatory reaction with proliferation of capillaries with further hemorrhages and progressive growth of the aneurysm. Some authors ${ }^{24}$ have proposed to call this pathologic process "aneurysm with intramural haematoma". Indirect signs of this are well visible on MR, in which the patent portion of the aneurysm is surrounded often asymmetrically by an area of inhomogeneous signal with a peripheral hyperintensity on T1-weighted images corresponding to the intramural clots of different ages. In these cases the ideal treatment should be the complete surgical excision of the lesion which, however, can pose complex technical problems. An alternative, whenever possible, is the occlusion of the aneurysm along with the parent vessel followed, if necessary, by steroid therapy. In our case the decision to for an endovascular approach was due to the consideration of the difficulty of surgery and the feasibility of endovascular treatment.

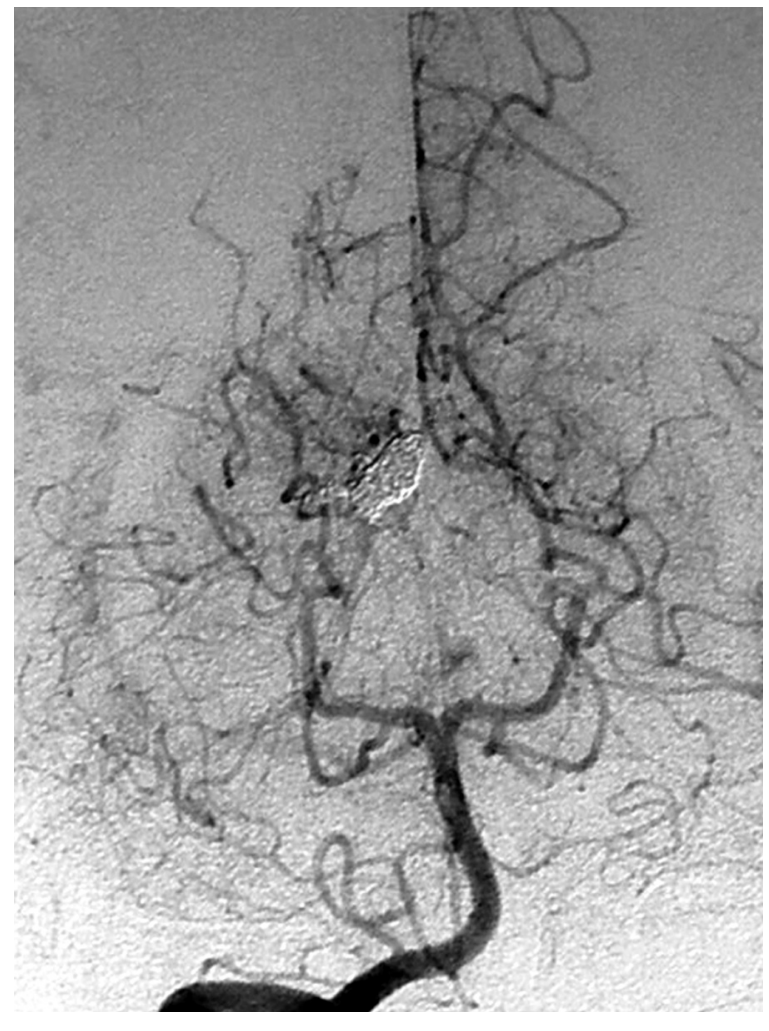

Figure 4 (A,B) Sagittal T1 and T2 weighted images six months later show disappearance of the aneurysm. Coils are recognizable. Control angiogram confirmed the complete exclusion of the aneurysm (C). 
In aneurysms of the peripheral segments (P3-P4) of the posterior cerebral artery the best treatment is the occlusion of the aneurysm along with the parent vessel.

This is particularly true in aneurysms thought to be dissecting to avoid possible further extension of the dissection This is commonly well tolerated ${ }^{25-27}$ due to the good leptomeningeal collateral circulation, particularly rich in children. Moreover the more important perforator branches supplying mesencephalon and thalamus arise from the more proximal segments (P1- P2) of the posterior cerebral

\section{References}

1 Heiskanen O: Ruptured intracranial arterial aneurysms of children and adolescents. Surgical and total management results. Childs Nerv Syst 5(2): 66-70, 1989.

2 Huang J, McGirt MJ et Al: Intracranial aneurysms in the pediatric population: case series and literature review. Surg Neurol 63(5): 424-432; discussion 432-433, 2005.

3 Lasjaunias P, Wuppalopati S et Al: Intracranial aneurysms in children aged under 15 years: review of 59 consecutive children with 75 aneurysms. Childs Nerv Syst 21: 437-450, 2005.

4 Meyer FB, Sundt M Jr et Al: Cerebral aneurysms in childhood and adolescence. J Neurosurg 70(3): 420425, 1989.

5 Ostergaard JR, Voldby B: Intracranial arterial aneurysms in children and adolescents. J Neurosurg 58(6): 832-837, 1983.

6 Roche JL, Choux M et Al: Intracranial arterial aneurysm in children. A cooperative study. Apropos of 43 cases. Neurochirurgie 34(4): 243-251, 1988. In French.

7 Laughlin S, TerBrugge KG et Al: Endovascular management of pediatric intracranial aneurysms. Interventional Neuroradiology 3: 205-214, 1997.

8 Proust F, Toussaint P et Al: Pediatric cerebral aneurysms. J. Neurosurg 94(5): 733-739, 2001.

9 Blackmore CC, Manourian AC: Acqueduct compression from venous angioma: MR findings.. Am J Neuroradiol 17: 458-460,1996.

10 Foran A, Donohue V et Al: Vein of Galen aneurysm malformation: closing the management loop. Ir Med J 97: 8-10, 2004.

11 Lasjaunias P: Vascular diseases in neonates infants and children. Springer Verlag. Berlin, Heidelberg, pp. 105120, 1997.

12 Jellinger G: Anatomopathology of non tumoral aqueductal stenosis. J Neurosurg Sci 30: 1-16, 1986.

13 Russel DS, Nevin S: Aneurysm of the great vein of Galen causing internal hydrocephalus: report of two cases. J Pathol Bacteriol 51: 447-448, 1940.

14 Crompton MR: The pathogenesis of cerebral aneurysms. Brain 89: 797-814, 1966.

15 Stehbens WE: Ultrastructure of Aneurysms. Arch Neurology 32: 798-807, 1975.

16 Yazbak PA, McComb JC et Al: Paediatric traumatic intracranial aneurysm. Pediatr Neurosurgery 223: 15-19, 1995.

17 Ventureyra ECG,Higgins MJ: Traumatic intracranial aneurysms in childhood adolescence.Case reports and artery and are therefore not involved in the treatment ${ }^{28,29}$.

\section{Conclusions}

Aneurysms in children are rare. They are frequently large and the presenting clinical symptoms can be due in many cases to mass effect with headache as the sole symptom. In the diagnosis, aneurysms, frequently dissecting, should be taken into account. Endovascular treatment whenever possible appears to be a good alternative to surgery. review of the literature. Child s Nerv Syst 10: 361-379, 1994.

18 Pasqualin A, Mazza C et Al: Intracranial aneurysms and subarachnoid hemorrhage in children and adolescents. Childs Nerv Sist 2: 185-190, 1986.

19 Fullerton HJ, Johnston S, Smith WS: Arterial dissection and stroke in children. Neurology 57: 1155-1160, 2001.

20 Massimi L, Moret J et Al: Dissecting giant vertebrobasilar aneurysms. Childs Nerv Syst 19: 204-210, 2003.

21 Schievink WI, Mokri B, Piepgras D: Spontaneous dissections of cervicocephalic arteries in childhood and adolescence. Neurology 44: 1607-1612, 1994.

22 Vilela P, Goul OA: Paediatric dissecting posterior cerebral aneurysms: report of two cases and review of the literature. Neuroradiology 48: 541-548, 2006.

23 Schubiger O,Valavanis A,Wichmann W: Growth mechanism of giant intracranial aneurysms; demonstration by CT and MR imaging. Neuroradionogy 29: 266-271, 1987.

24 Krings T, Alvarez $\mathrm{H}$ et Al: Growth and rupture Mechanism of partially Thrombosed Aneurysms. Interventional Neuroradiology 13: 117-126, 2007.

25 Bradač GB, Bergui M et Al: Periprocedural morbidity and mortality by endovascular treatment of cerebral aneurysms with GDC. A retrospective 12 years experience of a single centre. Neurosurg Review 30: 117-126, 2007.

26 Ciceri EF, Kluznik RP et Al: Aneurysms of the posterior cerebral artery: classification and endovascular treatment. Am J Neuroradiol 22: 27-34, 2001.

27 Hallacq P, Piotin M, Moret J: Endovascular occlusion of the posterior cerebral artery for the treatment of P2 segment aneurysms: Retrospective review of a 10 years series. Am J Neuroradiol 23: 1128-1136, 2002.

28 Zeal AA, Rhoton AL Jr: Microsurgical anatomy of the posterior cerebral artery. J Neurosurgery 48: 534-559, 1978.

29 Bradač GB, Daniele D et Al: Lacunes and other Holes: Diagnosis, Pathogenesis, Therapy. The Neuroradiology Journal 21: 35-52, 2008.
Gianni Boris Bradač, M.D.

Department of Neuroradiology University of Turin

Molinette Hospital, Via Cherasco, 15 10126, Torino, Italy 